\title{
Shared Decision Making in the Safety Net: Where Do We Go from Here?
}

\author{
Angelique B. Bouma, MD, Kristina Tiedje, PhD, Sara Poplau, BA, \\ Deborah H. Boehm, MPH, Nilay D. Shah, PhD, Matthew J. Commers, PhD, \\ Mark Linzer, MD, and Victor M. Montori, MD
}

Background: Shared decision making (SDM) is an interactive process between clinicians and patients in which both share information, deliberate together, and make clinical decisions. Clinics serving safety net patients face special challenges, including fewer resources and more challenging work environments. The use of SDM within safety net institutions has not been well studied.

Methods: We recruited a convenience sample of 15 safety net primary care clinicians (13 physicians, 2 nurse practitioners). Each answered a 9-item SDM questionnaire and participated in a semistructured interview. From the transcribed interviews and questionnaire data, we identified themes and suggestions for introducing SDM into a safety net environment.

Results: Clinicians reported only partially fulfilling the central components of SDM (sharing information, deliberating, and decision making). Most clinicians expressed interest in SDM by stating that they "selected a treatment option together" with patients (8 of 15 in strong or complete agreement), but only a minority ( 3 of 15) "thoroughly weighed the different treatment options" together with patients. Clinicians attributed this gap to many barriers, including time pressure, overwhelming visit content, patient preferences, and lack of available resources. All clinicians believed that lack of time made it difficult to practice SDM.

Conclusions: To increase use of SDM in the safety net, efficient SDM interventions designed for this environment, team care, and patient engagement in SDM will need further development. Future studies should focus on adapting SDM to safety net settings and determine whether SDM can reduce health care disparities. (J Am Board Fam Med 2014;27:292-294.)

Keywords: Patient Preference, Safety Net Clinics, Shared Decision Making

Shared decision making (SDM) occurs when clinicians and patients share information, deliberate jointly, and arrive at decisions that reflect patients' preferences. Clinician barriers to $\mathrm{SDM}^{1}$ may contribute to health disparities. Recent studies of African American patients demonstrate barriers even

This article was externally peer reviewed.

Submitted 3 September 2013; revised 4 December 2013; accepted 9 December 2013.

From the Department of Medicine (ABB, SP, DHB, ML), the Minneapolis Medical Research Foundation (DP, ML), and the Division of General Internal Medicine (DHB), Hennepin County Medical Center, Minneapolis, MN; the Department of Sociology and Anthropology, University of Lumière, Lyon, France (KT); the Department of International Health, CAPHRI School for Public Health and Primary Care, Maastricht University, Maastricht, Netherlands (ABB, MJC); Knowledge and Evaluation Research Unit, Department of Health Sciences Research, Mayo Clinic, Rochester, MN (NDS, VMM); and the Department of Medicine, University of Minnesota, Minneapolis, MN (ML). when patients seek to engage in information sharing. ${ }^{2}$ Clinics serving minority patients have more hectic workplaces and fewer resources, thus providing challenging settings for SDM. ${ }^{3}$ We studied perceptions of SDM among clinicians serving the urban poor. We sought to identify perceptions, barriers, and corrective steps to implement SDM in resource-constrained environments.

Funding: Support was provided, in part, by a Translating Information on Comparitive Effectiveness into Practice (TRICEP) grant, funded by Agency for Healthcare Research and Quality (AHRQ) grant 5R18HS018339-02 (ML, DHB, SP, NDS, VMM, KT).

Conflict of interest: none declared.

Corresponding author: Mark Linzer, MD, Division of General Internal Medicine, Hennepin County Medical Center, 701 Park Ave. (P7), Minneapolis, MN 55415 (E-mail: Mark. linzer@hcmed.org). 
Figure 1. Conceptual model for the shared decision-making process. (From Kriston et al, ${ }^{4}$ reprinted with permission of the publisher).

\begin{tabular}{|l|l|}
\multicolumn{1}{|c|}{ Theoretical key features } \\
$\begin{array}{l}\text { At least two parties (patient and physician) are } \\
\text { involved. }\end{array}$ \\
$\begin{array}{l}\text { Information is exchanged both ways. } \\
\text { Both parties are aware that treatment options } \\
\text { Both parties bring their decision criteria actively } \\
\text { and equally into the decision making process. }\end{array}$
\end{tabular}

\section{Methods}

This study was conducted with clinicians enrolled in a randomized trial testing SDM diabetes decision aids. Of 23 eligible clinicians (18 physicians and 5 nurse practitioners and physician assistants), we interviewed a convenience sample of 13 physicians and 2 nurse practitioners at Hennepin County Medical Center (HCMC). One clinician declined to participate. HCMC's patient population is primarily low income, with $65 \%$ of patients from communities of color and $25 \%$ from immigrant communities. The study took place from March to May 2011 and received local institutional review board approval. A conceptual framework of SDM (Figure 1) from Kriston et $\mathrm{al}^{4}$ structured our approach.

The SDM-Q-9 asks about SDM from the patient's perspective. ${ }^{4}$ We reframed the questions to be from the clinician's perspective. Response options included completely agree, strongly agree, somewhat agree, somewhat disagree, strongly disagree, and completely disagree. We also conducted interviews to explore the feasibility of SDM.

Audiotaped and transcribed interviews were reviewed by the lead author (ABB); a second reviewer assessed approximately $50 \%$ of the transcripts. A third reviewer (KT) assessed transcripts after coding was completed and agreed on the coding scheme. Themes were categorized into 3 domains: clinician barriers, patient barriers, and system barriers.

\section{Results}

Clinicians often made it clear to patients that a decision needed to be made (67\% strong or complete agreement) but less often (33\%) helped patients understand information, precisely explained treatment options $(33 \%)$, or thoroughly weighed treatment options with patients $(20 \%)$ (Table 1$)$.

Table 1. Findings of Shared Decision-Making Questionnaire from 15 Safety Net Clinician Respondents

\begin{tabular}{lc}
\hline & $\begin{array}{c}\text { Strongly or } \\
\text { Completely Agree } \\
(\mathrm{n}=15)\end{array}$ \\
\hline Statement & $73 \%$ \\
I told my patients there are different \\
options for treatment. \\
$\begin{array}{l}\text { My patients and I reached an agreement } \\
\text { on how to proceed. }\end{array}$ \\
$\begin{array}{l}\text { I made clear a decision needed to be } \\
\text { made. }\end{array}$ \\
$\begin{array}{l}\text { I asked my patients which option they } \\
\text { preferred. }\end{array}$ \\
$\begin{array}{l}\text { My patients and I selected a treatment } \\
\text { option together. }\end{array}$ \\
$\begin{array}{l}\text { I wanted to know how my patients } \\
\text { wanted to be involved. }\end{array}$ \\
$\begin{array}{l}\text { I explained the advantages and } \\
\text { disadvantages of treatment options. }\end{array}$ \\
$\begin{array}{l}\text { I helped my patients understand all the } \\
\text { information. }\end{array}$ \\
$\begin{array}{l}\text { My patients and I thoroughly weighed } \\
\text { the treatment options. }\end{array}$ \\
\hline
\end{tabular}


Time pressure was noted by all clinicians: "If I did all these steps, I would never get through the day." Other clinician issues included practice preferences ("I have a clear bias I'm sure comes across [in] the way I present the data"), and visit content ("It's a lot to try to get the patient to absorb in a 15-minute visit...").

Clinicians also identified patient-related barriers, such as willingness to participate and cultural differences. System barriers included resources and organizational culture ("A place where every point of care is aimed around SDM lets everybody do their job ..., but if it feels counter-cultural ..., then it's much harder ...").

\section{Discussion}

Our safety net primary care clinicians express interest in SDM but do not often practice it. Barriers include time available, personal preferences, knowledge, and training. There are also concerns about patients' willingness to share decisions and system factors, including lack of resources and organizational culture.

Our work adds to that of the systematic review by Légaré et $\mathrm{al}^{1}$ and may be the first to focus on SDM adoption in safety net care. Our results show that physician-identified barriers to SDM may be of similar nature throughout the health care system. What may distinguish barriers in safety net facilities is their magnitude, the importance of the context of the facility and patients, and the efforts needed to overcome them. Results from ongoing trials of SDM implementation at HCMC may offer more insights into the effect these barriers may have on the efficacy of such interventions.
Limitations of our study include the single study site and the limited analysis of qualitative data. Action steps to increase the use of SDM in safety net settings include (1) developing efficient SDM protocols using decision aids; (2) training clinicians in issues related to cultural and context awareness, health literacy, and SDM; (3) promoting patient interest in SDM with health coaches; and (4) developing team cohesion and a culture that supports SDM. As Nowakowski et $\mathrm{al}^{5}$ suggest, we can learn from "exemplary providers who overcome contextual barriers" and actively participate in SDM with their patients.

\section{References}

1. Légaré F, Ratté S, Gravel K, Graham ID. Barriers and facilitators to implementing shared decision making in clinical practice: update of a systematic review of health professionals' perceptions. Patient Educ Couns 2008;73:526-35.

2. Peek ME, Tang H, Cargill A, Chin MH. Are there racial differences in patients' shared decision making preferences and behaviors among patients with diabetes? Med Decis Making 2011;31:422-31.

3. Varkey AB, Manwell LB, Williams ES, et al; MEMO Investigators. Separate and unequal: clinics where minority and nonminority patients receive primary care. Arch Intern Med 2009;169:243-50.

4. Kriston L, Scholl I, Hölzel L, Simon D, Loh A, Härter M. The 9-item Shared Decision Making Questionnaire (SDM-Q-9). Development and psychometric properties in a primary care sample. $\mathrm{Pa}-$ tient Educ Couns 2010;80:94-9.

5. Nowakowski KE, Tilburt JC, Kaur JS. Shared decision making in cancer screening and treatment decisions for American Indian and Alaska Native communities: can we ethically calibrate interventions to patients' values? J Canc Educ 2012;27:790-2. 